\title{
Intramuscular Lipoma
}

National Cancer Institute

\section{Source}

National Cancer Institute. Intramuscular Lipoma. NCI Thesaurus. Code C7450.

A benign tumor, composed of mature adipocytes, that arises within skeletal muscle fibers. 